Cahiers de recherches médiévales

Journal of medieval studies

$8 \mid 2001$

La protection spirituelle au Moyen Âge

\title{
Une apparition pour protéger
}

Le manteau de la Vierge au XIII ${ }^{\mathrm{e}}$ siècle

\section{Sylvie Barnay}

\section{OpenEdition}

Journals

Édition électronique

URL : https://journals.openedition.org/crm/378

DOI : $10.4000 / \mathrm{crm} .378$

ISSN : 1955-2424

Éditeur

Honoré Champion

\section{Édition imprimée}

Date de publication : 15 janvier 2001

Pagination : 13-22

ISSN : 1272-9752

\section{Référence électronique}

Sylvie Barnay, "Une apparition pour protéger », Cahiers de recherches médiévales [En ligne], 8| 2001, mis en ligne le 13 mars 2008, consulté le 15 décembre 2022. URL : http://journals.openedition.org/ crm/378; DOI : https://doi.org/10.4000/crm.378

Ce document a été généré automatiquement le 15 décembre 2022.

Tous droits réservés 


\title{
Une apparition pour protéger
}

Le manteau de la Vierge au XIII ${ }^{\mathrm{e}}$ siècle

\section{Sylvie Barnay}

\begin{abstract}
« La Vierge est notre protectrice, notre gonfalonière ;
Elle défend tous ceux qui veulent se grouper autour d'elle ;

Contre nos ennemis, elle est très forte ennemie ;

Bienheureux l'homme et la femme qui sont sous sa vêture.

Elle est notre refuge si l'on veut se tourner vers elle :

Chaque homme qui pèche, elle se met à le protéger,

S'il veut seulement être repentant et veut faire amende,

Et s'il veut grandement se recommander à elle [...] $»^{1}$
\end{abstract}

1 Dans ses «Louanges à Marie » composées à la fin du XIII ${ }^{\mathrm{e}}$ siècle, le poète milanais Bonsevin dalla Riva ( $†$ vers 1315) chante la protection de la Vierge dans des termes qui résument la pensée du Moyen Âge central sur la médiation mariale. Les textes visionnaires en offrent une semblable définition. Les récits de visions, d'apparitions et de révélations de la Mère de Dieu ont en effet servi de cadre narratif et conceptuel à une déclinaison du système de protection spirituelle tel qu'il fut pensé par les hommes médiévaux. Le langage visionnaire témoigne de cette élaboration. Il définit un espace de sécurité "entre ciel et terre ", ici-bas et au-delà, où la Mère de Dieu occupe une fonction majeure. Tout un réseau de métaphores raconte cette médiation protectrice à l'œuvre. Au nombre de celles-ci figure notamment la métaphore du manteau marial. Les historiens, depuis Paul Perdrizet jusqu'à Jean Delumeau, ont bien mis en valeur les représentations symboliques qui lui sont liées ${ }^{2}$. Dans l'optique définie par ce cahier de recherches, cet article s'inspire à son tour de la synthèse de Jean Delumeau « Rassurer et protéger». En reprenant le dossier thématique bien connu de la «Vierge au manteau ", il cherche à souligner le sens spirituel donné au vêtement protecteur de Marie dans les récits visionnaires du XIII ${ }^{\mathrm{e}}$ siècle. 


\section{Le manteau de Marie}

\section{Vêtement incorruptible}

2 Une première transposition doctrinale apparait derrière l'image du manteau protecteur. Elle trouve sa source dans le fondement même de la croyance aux apparitions mariales attestée dès la fin du IV ${ }^{\mathrm{e}}$ siècle à Constantinople ${ }^{3}$. La base de la croyance n'est en effet pas séparable du principe qui gouverne la pensée chrétienne des pères grecs de la fin de l'antiquité tardive : la chute a fait perdre à l'homme sa place originelle et l'Incarnation du Christ a rétabli la voie qui conduit au salut. La Chute a séparé la créature de son Créateur, précipitant les hommes du paradis originel vers un monde opaque à la vision de Dieu. Avant la chute, l'homme captait la vision de Dieu au moyen du miroir de son âme. Mais la chute, en alourdissant la matière, a voilé la surface du miroir des créatures, le mettant dans l'incapacité de refléter la lumière divine. Elle a fait perdre à l'homme sa qualité de voyant de Dieu. La vision de la Vierge joue un rôle dans cette restauration. Dès les débuts de la croyance, elle est présentée comme le miroir qui permet aux hommes d'accéder à la vision divine ${ }^{4}$. La Vierge a permis la venue du Christ sur la terre. Par sa coopération à l'Incarnation, elle a en effet rendu possible la restauration du chemin reliant les hommes à Dieu. Par sa médiation, a été rétablie la communication entre la créature et son Créateur. Le rôle médiateur de la Vierge dans la réconciliation entre les hommes et Dieu explique alors le rôle prêté à ses visions et notamment à son manteau dans cette opération.

3 Toute une exploration narrative et exégétique est ensuite à l'origine de récits où la Vierge "habille", recouvre et protège les hommes médiévaux avec son vêtement céleste. Il est important de rappeler, dans un premier temps, les principales étapes de cette exploration pour comprendre le sens de l'exploitation du motif visionnaire de la Vierge au manteau au XIII ${ }^{e}$ siècle ${ }^{5}$. Dès la fin du $\mathrm{VI}^{\mathrm{e}}$ siècle, la liturgie byzantine condense en une image saisissante l'équivalence qui est formulée entre l'habit protecteur de Marie et le corps du Christ: "À tous les fidèles comme un vêtement d'incorruptibilité, tu as donné ton saint habit avec lequel tu abrites le saint corps du Christ, ô toi qui es l'abri protecteur et divin des hommes $»^{6}$. Cette thématique est traduite dans le registre visionnaire de manière synchronique. Recopiant en partie un chroniqueur byzantin contemporain, Grégoire de Tours ( $†$ 594) rapporte ainsi dans ses Libri miraculorum le récit d'une protection miraculeuse d'un enfant juif jeté dans le feu après avoir reçu le corps du Christ ${ }^{7}$. Au milieu des flammes, l'enfant raconte qu'il a vu une dame semblable à celle de son image lui apparaître et le recouvrir de son manteau protecteur8. L'homme d'Église atteste ainsi la circulation du récit dans l'aire méditerranéenne. L'intrigue même du miracle s'inspire de l'histoire biblique des trois juifs jetés dans la fournaise sur l'ordre du roi Nabuchodonosor et sauvés par un ange (Dn 3, 24-50), mais l'ange de la vision biblique a fait place à Marie dans la vision du miracle. La grande vénération dans laquelle les Grecs tenaient les reliques du voile et du manteau de la Vierge aux vertus protectrices transparaît à travers ce récit qui sert également de miroir aux joutes doctrinales entre juifs et chrétiens.

Le motif du manteau protecteur de Marie appartient dès lors au premier fonds de la mariophanie du Haut Moyen Âge. Il circule ensuite dans l'hagiographie byzantine comme en témoigne, par exemple, la Vie de saint André le Fol composée par Nicéphore dans la première moitié du $\mathrm{X}^{\mathrm{e}}$ siècle : «André vit nettement de ses yeux une Dame, de 
stature très élevée, s'avancer dans sa parure féminine, hors des portes royales, environnée d'un cortège harmonieux [...]. La prière terminée, elle s'approcha du sanctuaire, recommença à prier pour le peuple qui l'entourait. Alors, le voile étincelant, elle l'écarta, et, le déployant avec une majesté imposante, elle le maintint étendu de ses mains sans tache, elle en couvrit tout le peuple qui se tenait au-dessous. Et durant un temps assez considérable, ces hommes admirables, Épiphane et André, le contemplèrent, déroulé au-dessus de la foule et laissant rayonner tout autour une gloire divine, à la manière de l'électrum ${ }^{9}$. En Occident, il faut attendre le XI ${ }^{\mathrm{e}}$ siècle pour que la littérature latine des miracles de la Vierge donne à nouveau réception au motif visionnaire au moment où les mentalités, redécouvrant l'humanité du Christ, découvrent aussi celle de sa Mère et lancent véritablement le culte marial en Occident.

\section{Vêtement d'Incarnation}

5 L'exploration exégétique du XIII ${ }^{\mathrm{e}}$ siècle mène à son terme le parallélisme déjà opéré entre le vêtement de Marie et le corps du Christ par les liturgistes byzantins. Depuis les années 1150 , le sein virginal de Marie est comparé à un vestiaire où le Christ se revêt du vêtement d'Incarnation. À la suite des exégètes cisterciens, le De Laudibus Beatae Mariae Virginis attribué à Albert le Grand ( $† 1280)$ compare de manière explicite l'utérus de Marie à une garde-robe : « De même, le vestiaire de cette chambre à coucher, en langue gallicane « la garde-robe » est proprement dit l'utérus virginal dans lequel le grand prêtre (le Christ) a revêtu les vêtements de la plus pure des chairs ${ }^{10}$. L'image est audacieuse pour dire que Dieu a pris dans le vestiaire marial la robe de son humanité. Le vêtement de Marie est donc le vêtement de l'Incarnation ${ }^{11}$. En amont du texte albertinien, saint Bonaventure ( $\dagger 1274)$ rappelle à son tour que le Christ-prêtre revêt sa robe rouge et blanche dans le tabernacle de l'utérus virginal et marial ${ }^{12}$. Le sein de la Vierge où le Christ s'est habillé de chair est semblable à la sacristie dans lequel le prêtre met son aube sacrée pour dire la messe. Les liturgistes font au même moment revêtir au Christ le vêtement d'Incarnation dans le sein de Marie. Le Rational de Guillaume Durand de Mende († 1296) compare ainsi la sacristie dans laquelle le prêtre revêt les vêtements sacrés au sein de la Vierge où le Christ s'est revêtu du saint vêtement de sa chair ${ }^{13}$. Dans la théologie du XIII ${ }^{e}$ siècle, la Vierge est en effet considérée comme la source du sacerdoce du Christ et du sacerdoce des prêtres, la Mère de la prêtrise ${ }^{14}$. Le manteau marial devient alors la métaphore du corps du Christ.

\section{Vêtement d'Église}

Consacrant à la "largitas et misericordia Mariae» tout un passage de son De Laudibus Beatae Mariae Virginis, Albert le Grand résume encore le sens prêté cette fois à la dimension du manteau de la Vierge ${ }^{15}$. Son amplitude est semblable au ciel. Il est vaste comme la patrie céleste. Il a en effet la triple forme du corps du Christ, corps d'Incarnation, corps eucharistique et corps ecclésial ${ }^{16}$. Le jeu des métaphores bibliques se prête ensuite dans le même corpus à toute une déclinaison exégétique qui permet d'entrevoir le sens des mensurations du manteau de la Bienheureuse Mère de Dieu. Sous la plume du dominicain, la Vierge est en effet devenue « Marie ciel » et «Marie soleil », « Marie nuage » et « Marie firmament ». Elle est encore «Marie habitation » et "Marie tabernacle » et "Marie arche », " Marie cellule» et "Marie temple », " Marie cité de Dieu» et "Marie Église $»^{17}$... Autant de formes données au Christ, autant de 
formes données à la Mère de Dieu. Marie est en effet également définie par les théologiens comme "la Maison de Dieu», le "temple de Dieu», le "tabernacle de Dieu », l'« arche de Dieu »... puisqu'elle est le miroir de Dieu. À la fin du XIII siècle, à elle seule, Marie est devenue la forme de toutes les formes, le corps de tous les corps puisqu'elle est à la fois comparée à l'univers et à l'Église, à l'infiniment petit et à l'infiniment grand. Elle englobe toutes ces formes à la manière d'une poupée gigogne laissant découvrir des formes similaires de plus en plus petites - depuis l'univers jusqu'à la cellule du frère dominicain -, chacune d'elle étant à son échelle, l'expression imagée de la Création de Dieu. Au siècle où l'exégèse définit cette construction vertigineuse, le manteau de Marie devient alors lui-même dans les récits visionnaires le « corps » d'un corps d'Église à part entière : celui des ordres religieux.

\section{Robe de protection}

\section{Corps des ordres religieux}

7 En même temps qu'il donne une formulation exégétique du sein de la Vierge comme corps du Christ, le XIII ${ }^{e}$ siècle multiplie les récits de visions et d'apparitions où la Reine du ciel recouvre les ordres religieux de son manteau pour les réintroduire dans un ordre semblable à celui d'avant le péché. En Occident, comme l'a démontré Jean Delumeau, l'ordre cistercien est le premier à relancer la tradition narrative de la Vierge au mantelet protecteur, synonyme à la fois d'habitation protectrice et de corps d'Incarnation. Le Dialogus miraculorum commencé en 1217 à l'initiative de Césaire d'Heisterbach (†1240) alors maître des novices, rappelle ainsi à l'aide d'exempla significatifs, l'identité entre la robe mariale et la tunique du Christ. Les vêtements des moines sont eux-mêmes tissés à leur ressemblance ${ }^{18}$. Le recueil d'édification montre que les générations monastiques sont abritées et protégées sous le manteau de Marie qui désigne aussi la robe du Christ. À tel de ces moines exemplaires inquiet de ne pas apercevoir ses frères dans le giron marial, la Vierge se charge ainsi de répondre : «Ceux de Cîteaux me sont au contraire si chers et si familiers que je les réchauffe sous mes bras. Et ouvrant le manteau qui la couvrait et qui était d'une largeur merveilleuse, elle lui montra une multitude innombrable de moines, de frères convers et de nonnes ${ }^{19}$. La familiarité signale usuellement un degré de positionnement avancé dans l'ordre de la parenté spirituelle ${ }^{20}$. L'emploi du terme est donc ici loin d'être anecdotique et cherche à manifester la filiation divine qui unit l'ordre de Cîteaux à la Vierge Marie représentée comme la Mère spirituelle de l'ordre. Le manteau épiphanise ainsi, en quelque sorte, sa maternité divine.

8 En laissant entrevoir les moines cisterciens sous la robe mariale, le récit cherche en effet également à raconter de manière imagée, le mouvement qui réintroduit déjà les moines cisterciens dans une Création "sans désordre ", à un moment où Cîteaux connaît précisément une crise importante ${ }^{21}$. Le thème trouve un antécédent dans le pont que le langage visionnaire établit entre les hiérarchies célestes et les hiérarchies terrestres qui structurent l'ordre de l'univers selon l'idéologie des ordres héritière du Pseudo-Denys ${ }^{22}$. Dès le milieu du $\mathrm{X}^{\mathrm{e}}$ siècle, recevoir un vêtement céleste des mains de la Vierge Marie est perçu comme le signe d'un avancement dans les étages de la hiérarchie, de l'ordre terrestre vers l'ordre céleste ${ }^{23}$. Ce don assigne leurs bénéficiaires à prendre le premier rang dans l'ordre des hiérarchies divines, comme le répètent 
encore les collections de miracles de la Vierge aux $\mathrm{XI}^{\mathrm{e}}$-XII ${ }^{\mathrm{e}}$ siècles qui font circuler ce type de motif ${ }^{24}$. Entrer sous le manteau protecteur de la Vierge ou recevoir de ses mains une vêture divine tirée de sa propre garde-robe devient alors le gage d'une élection céleste garante de la béatitude promise aux bienheureux.

\section{Giron de maternité spirituelle}

9 À l'imitation de Cîteaux, les ordres religieux mendiants nés au XIII ${ }^{e}$ siècle ont également trouvé dans le manteau protecteur une manière de dire et de décrire à la fois leur origine et leur fin, leur filiation divine et leur élévation spirituelle. L'espace narratif offert par la mariophanie a continué de se prêter à la course à ce double enjeu, reflet de la concurrence assidue que se livrent les ordres monastiques et mendiants au lendemain du Concile de Latran (1215). Jourdain de Saxe ( $†$ 1237), le premier biographe du fondateur de l'ordre dominicain, reprend à son tour le récit dont la forme narrative, par définition "vierge", se prête à toutes les actualisations. L'hagiographe raconte également dans son Libellus de principiis ordinis praedicatorum le don marial de l'habit de l'ordre dominicain au frère Réginald d'Orléans ${ }^{25}$. Un demi-siècle plus tard, Thierry d'Apolda, rédigeant entre 1280 et 1291 le libelle de la Vie de saint Dominique ( $† 1221)$, déplace à l'avantage du fondateur de l'ordre la vision mariale du don du vêtement. Sur la base des notices qui servent de source aux Miracula Beati Dominici, il reprend le motif désormais bien connu du manteau de la Vierge dévoilant l'ordre religieux sous sa vêture originelle. Ravi en esprit, saint Dominique voit le Seigneur et sa Mère revêtue d'un magnifique manteau couleur saphyr. Alors que sous le giron de la Vierge, se pressent «les fondateurs d'ordre ayant enfanté au Christ des fils et des filles selon l'esprit ", Dominique ne voyant nulle part ses propres fils se met à pleurer. Ouvrant son large manteau, la Vierge lui découvre alors « sous son sûr abri » les frères de l'ordre dominicain ${ }^{26}$.

10 La surenchère spirituelle fonctionne alors à plein sur le terrain de la filiation divine et de la maternité spirituelle de Marie que les nouveaux ordres revendiquent ${ }^{27}$. Thomas de Cantimpré († 1270/72), compilant la littérature exemplaire primitive de l'ordre, place ainsi ces paroles dans la bouche de la Vierge apparue à un cistercien: "J'ai d'autres frères que je favorise de mon patronage et que j'enveloppe de ma protection ». Ouvrant le manteau où se trouvent blottis les frères prêcheurs, elle ajoute: "Voici ceux qui tâchent tout spécialement que le sang de mon fils chéri n'ait pas été versé en vain ${ }^{28}$.

11 L'hagiographie franciscaine formule le patronage marial de l'ordre en des termes équivalents. Dans la seconde Vita de saint François qu'il compose en 1248, Thomas de Célano écrit, par exemple, du Poverello qu'«il voulut la [Marie] choisir comme patronne de l'ordre et mettre sous ses ailes pour qu'elle les couve et les protège jusqu'à la fin, les frères que lui-même un jour devrait quitter $~_{29}$. L'affirmation doctrinale ne trouve pas immédiatement une adaptation visionnaire. C'est seulement à la fin du $\mathrm{XIV}^{\mathrm{e}}$ siècle que la Legenda de saint François due à la plume de Bernardin de Besse introduit le motif visionnaire de la Vierge au manteau protecteur dans la tradition narrative de l'ordre ${ }^{30}$. L'image a-t-elle suppléé ici à la vision? Il n'est pas improbable d'avancer que l'émergence de la première iconographie occidentale de la Vierge au manteau dans un climat franciscain en 1267 ait pu jouer en faveur de l'éclipse narrative de l'apparition de la Vierge au manteau ${ }^{31}$. Les images de la Vierge dite de miséricorde se répandent en effet rapidement par l'intermédiaire des confréries franciscaines qui la 
portent en effigie sur leurs bannières. Mais le motif de la Vierge au manteau protecteur était désormais définitivement entré dans les formulations de la mémoire des origines des ordres. Au tout début du XIV e siècle, la tradition de l'ordre des Servites, suivie ultérieurement par la tradition de l'ordre des Carmes, en fournissent encore la manifestation tangible ${ }^{32}$. Les ordres ne cessent de continuer de rivaliser par visions mariales interposées pour occuper la première place sous le manteau de Marie, celle qui les plaçait au plus haut, aussi bien sur la terre que dans le ciel.

\section{Enceinte de salut}

Pour les mêmes raisons, le manteau marial s'ouvre alors à tous les corps de la société, qu'ils soient religieux ou laïcs. Tandis que la Vierge «se confond avec l'Ecclesia et se spatialise comme patronne", elle accueille sous sa grande robe toute l'humanité en demande de salut ${ }^{33}$. Délivrer l'humanité de sa souffrance, la réconcilier avec Dieu, la protéger des malheurs et du péché, c'est en effet aussi ce que le siècle continue de demander à la Reine du Ciel porteuse du vêtement de miséricorde. Les recueils de miracles de la Vierge du XIII ${ }^{\mathrm{e}}$ siècle s'en font notamment l'écho. Dans ce type de récits édifiants, la Vierge sauve alors tous les "corps" pensés alors comme des miroirs métaphoriques $\mathrm{du}$ «corps du Christ : corps des hommes, corps des villes, corps des confréries...

13 Le thème connaît donc un développement sans fin à mesure que les différents " corps ", qu'ils soient religieux ou sociaux, ont besoin d'être protégés. Au XIII ${ }^{e}$ siècle, les corps des cités reçoivent par exemple pour la première fois une protection du divin manteau de la Vierge. Les Miracles de la Vierge du bénédictin Gautier de Coincy ( $† 1236)$ évoquent ainsi l'épopée de la Vierge protectrice de Constantinople recouvrant la cité de son manteau: "Cele Royne glorieuse/Cele grande dame merveilleuse/En son manteau reçoit les cous/Et rebondit la pière entre eus $»^{34}$. Jusque-là le motif s'était arrêté à décrire l'apparition de la Vierge sur les remparts sans faire intervenir le curseur narratif du manteau protecteur ${ }^{35}$. Par l'intermédiaire du manteau de Marie, le corps de la cité peut donc "faire corps » avec le corps du Christ, c'est-à-dire à sa manière intégrer une idéologie des ordres duquel, initialement, le monde urbain se trouvait exclu $^{36}$. Le corps de la cité englobé sous le corps/manteau de Marie «fait corps » avec l'Église. La mariophanie fonctionne à alors à plein comme outil de normalisation religieuse. Le XIII ${ }^{e}$ siècle est le siècle des " apparitions modèles " "

C'est pourquoi, le manteau marial couvre encore par exemple de son manteau le corps des naufragés pour les conduire au rivage ou le corps des femmes enceintes pour leur permettre d'accoucher. Il en est ainsi dans ce récit anonyme : « Une femme grosse était allée au Mont Saint-Michel. Surprise par la marée montante, elle invoqua la protection de la bienheureuse Vierge. Alors et à la vue de tous, cette femme vit la Bienheureuse Vierge étendre sur elle la manche de son vêtement et la préserver ainsi du bruit et $\mathrm{du}$ choc de la mer. La plus petite goutte d'eau ne put même atteindre ses habits. Ce fut ainsi que, comme dans le lit le plus tranquille, elle mit au monde un fils, en jouissant des consolations de la Bienheureuse Marie jusqu'à ce que les flots s'étant retirés, eussent laissé le chemin libre à la mère et à l'enfant $»^{38}$.

Aux femmes des miracles en passe d'accoucher dans la souffrance, la Vierge donne en effet son enveloppe de femme qui enfante sans douleur, à l'image de la femme du MontSaint-Michel... La sainte Mère de Dieu procure en effet des robes de ressemblance aux 
femmes qui l'imitent. Elle habille d'une enveloppe de virginité les femmes visionnaires de Dieu engagées dans le chemin de l'imitation mariale ${ }^{39}$. La Vierge revêt également les saints des vitae et des miracula d'un vêtement d'attente tissé de chair et de lumière et non plus tunique de chair et de péché. La Bible a donné la liste des costumes autorisés pour entrer aux cieux : la tenue de noce de la parabole évangélique; l'habit blanc de l'Apocalypse destiné à masquer la nudité de l'homme, son vêtement de chair qui l'habille depuis la chute $(\mathrm{Ap} \mathrm{3,18)}$; la robe blanche de l'attente de la fin des temps tirée encore du vestiaire de l'Apocalypse $(A p 6,11)$ etc. La Vierge puise à cette même penderie pour vêtir les hommes spirituels qui accèdent à sa divine vision.

Comme la femme des Évangiles qui touche la frange du manteau de Jésus pour être sauvée (Mt 9, 20 ; Mc 5, 21-43; Lc 8, 40-56), les visionnaires du XIII ${ }^{e}$ siècle cherchent pareillement à toucher le bord du manteau des apparitions de la Vierge pour être sauvés. L'hagiographe de la converse camaldule Gérardesca de Pise (†1260) en donne un exemple emblématique parmi tant d'autres, rapportant une vision de la sainte qui « vit [son ami] religieux tirer les bords du vêtement de Notre-Dame en toute dévotion [...]. La Vierge étendit ensuite le manteau dont elle était vêtue et elle reçut le religieux sous celui-ci ${ }^{40}$. Le manteau, dont Gérardesca affirme encore qu'il est «un étonnant mantelet tourbillonnant » enroulant dans sa ronde spiralée toute la Création, s'ouvre ainsi pour quiconque désire réintégrer l'ordre divin de la Création ${ }^{41}$. Alors la Dame de Beauté ouvre sa robe et accueille l'âme désireuse de se réfugier sous sa protection ou sous sa direction. Tous les hommes qui cherchent à entrer sous le manteau de Marie sont en effet invités à prendre place dans l'univers ordonné de la Création. Nombre de récits visionnaires du XIII ${ }^{e}$ siècle font de ce positionnement un indice de sainteté. Pour contrer les attaques calomnieuses qui accusent le frère dominicain Barthelemy de Trente († 1251) d'entretenir un commerce douteux avec le négoce du monde, son hagiographe laisse par exemple sciemment profiler sa silhouette sous le manteau de la Vierge. Alors qu'une sœur nommée Mabilia implore la Vierge pour le salut du frère, Marie se tient en riant devant elle et étendant le manteau dont elle est revêtue, montre le saint homme vêtu de l'habit de son ordre en disant : «Il est tel que tu le vois ici, là où il $e s t »^{42}$. La place que le frère tient sous le manteau céleste est en effet le miroir de celle qu'il occupe dans le monde et dont la Vierge est la garante et protectrice de la divine ordonnance...

Il existe ainsi autant de récits au manteau de la Vierge que de traductions pour dire et décrire le mouvement même qui conduit les hommes médiévaux de la Chute à la Rédemption. En formulant une stricte parenté entre «la Vierge comme vêtement du Christ » et "le Christ comme robe de Marie », le langage exégétique du XIII siècle a posé les véritables conditions d'une transposition visionnaire de cette homologie. Dès lors, entrer sous le manteau de Marie a aussi signifié "revêtir le Christ» (Rm.13, 14; Ga. 3, 27). On comprend alors la fortune tant narrative qu'iconographique du thème au $\mathrm{XIII}^{\mathrm{e}}$ siècle dont Jean Delumeau a montré qu'elle allait de pair au XIV siècle avec le développement des confréries ${ }^{43}$. Jouant principalement de la définition métaphorique du corps du Christ comme corps d'Incarnation et comme corps d'Église, les auteurs ont alors déployé la robe mariale selon le prodigieux angle d'ouverture qui était le leur. Le manteau de la Vierge est ainsi devenu synonyme d'un "corps" idéal où pouvait prendre place tous ceux qui franchissaient l'enceinte du péché pour entrer dans le sein de l'Église. C'est alors que ce manteau de Marie pouvait protéger, c'est-à-dire contrôler 
en même temps qu'introduire les hommes dans la lumière du Christ Sauveur et Rédempteur.

\section{NOTES}

1. Cité par H. Spitzmuller, Poésie italienne du Moyen Âge, XII ${ }^{e}-X V^{e}$ siècle, Paris, 1971, pp. 433-434 et J. Delumeau, Rassurer et protéger. Le sentiment de sécurité dans l'Occident d'autrefois, Paris, Fayard, 1989, p. 265.

2. H. Belting, Image et culte. Une histoire de l'art avant l'époque de l'art, Paris, Cerf, 1998 ; H. BeltingIhm, Sub matris tutela, Heidelberg, 1976 ; J. Delumeau, Rassurer et protéger, op. cit.; V. JenshSussmann, Vorlaüfer und Varienten des Mittelälterliche Schutzmantelbild,Université de Marbourg, 1954 (dactylographié) ; V. Jensh-Sussmann, « Maria mit dem Schutzmantel », Marburger Jahrbuch für Kunstwissenschaft,5, 1929, pp. 285-351; G. Schiller, s.v. "Die Schutzmantelmadonna", Ikonographie der christlichen Kunst, t. IV, 2 ; M. Heizmann, La Vierge au manteau de protection. Etude d'un thème iconographique, Metz, 1983; P. Perdrizet, La Vierge de miséricorde, Paris, 1908. De manière secondaire, voir aussi W. Deonna, "La Vierge de miséricorde », Revue de l'histoire des religions, 1916, pp. 189-227 ; E. Mâle, L'art religieux de la fin du Moyen Âge, Paris, Armand Colin, 1925, pp. 198-209 ; M. Volberg, La Vierge, notre médiatrice, Grenoble, 1938.

3. Nous nous permettons de renvoyer à S. Barnay, Le ciel sur la terre. Les apparitions de la Vierge au Moyen Âge (préface de Jean Delumeau), Paris, Cerf, 1999, pp. 25-35.

4. Ibid.

5. Néologisme forgé sur le terme de christophanie.

6. E. Sendler, Les icônes byzantines de la Mère de Dieu,Paris, Desclée de Brouwer, 1992, p. 113. Sur la protection mariale à Byzance, voir surtout E. Follieri, « La Theotokos difesa del popolo cristiano nelle tradizioni bizantine et italogreche », Mélanges René Laurentin, Paris, 1990, pp. 377-383.

7. Gregorii episcopi Turonensis libri miraculorum, liber I : De gloria martyrum, P.L., vol. 71, col. 714-715.

8. Sur les reliques et le manteau de la Vierge vénérés à Constantinople, A. Cameron, "The Theotokos in sixth century Constantinople », The Journal of Theological Studies, 29-1978, pp. 79-108.

9. Vita S. Andreae Sali, P.G., vol. 111, col. 848-849.

10. B. Alberti magni opera omnia. De laudibus B. Mariae Virginis libri XII, éd. de A. Borgnet, Paris, 1898, p. 512 : Item, vestiarum hujus thalami, gallice, la garde robe, uterus proprie virginalis, in quo summus pontifex induit vestes mundissimae carnis ubi dicitur quod in arca vestimenta complicantur.

11. Sur les rapports entre exégèse et récit visionnaire, nous nous permettons également de renvoyer à notre thèse à paraître : Un moment vécu d'éternité. Les apparitions de la Vierge (XII ${ }^{e_{-}}$ $X V^{e}$ siècles).

12. Decem opuscula ad theologiam mysticam spectantia, Quaracchi, 1965, t. IX, p. 672.

13. Rational ou manuel des divins offices, éd. et trad. C. Barthélémy, Paris, 1854, p. 85, cité par J. Wirth, «L'apparition du surnaturel dans l'art du Moyen Âge », L'image et la production du sacré, Paris, Klincksieck, 1991, p. 152.

14. R. Laurentin, « Marie et la messe. Essai sur un problème de spiritualité sacerdotale ", Nouvelle Revue théologique, 1949, pp. 39-49.

15. B. Alberti magni opera omnia. De laudibus B. Mariae Virginis libri XII, op. cit.,pp. 66-67.

16. Ibid.

17. Ibid. 
18. Césaire d'Heisterbach, Dialogus miraculorum, éd. J. Strange, distinctio septima, cap. XVI, p. 23. H. Hilka, Die wundergeschichten des Caesarius von Heisterbach, Bonn, 1933, p. 158, n 231 (par exemple). Bonne présentation du Dialogus miraculorum dans F. Wagner, «Studium zu Caesarius von Heisterbach ", Analecta Cisterciensia, 29, 1975, pp. 79-95.

19. Césaire d'Heisterbach, Dialogus miraculorum, Op. cit., distinctio septima, cap. XVI, p. 59.

20. Nous avons tenté de démontrer ce point notamment dans un article intitulé « Lactations et apparitions de la Vierge. Une relecture de la règle, une lecture de la vie de saint Bernard", Unanimité et diversité cisterciennes (IV ${ }^{\mathrm{e}}$ colloque international du CERCOR, Dijon, 23-25 septembre 1998), Publications de l'Université de Saint-Étienne, 2000, pp. 161-174. Nous renvoyons également à la bibliographie donnée dans ce cadre.

21. Juste avant le Concile de Latran IV (1215), le pape Innocent III a menacé de faire disparaître l'ordre de Cîteaux s'il ne mettait pas fin aux relâchements observés à l'encontre des statuts primitifs de l'ordre. Cîteaux s'éloignait alors de son idéal primitif.

22. S. Barnay, Le ciel sur la terre, op. cit., chapitre I.

23. C'est ce que traduit par exemple la vision de l'évêque Ildefonse de Tolède dont la première version est due à l'évêque Cixila († 932), Corpus scriptorum muzarabicorum, éd. I. Gil, Madrid, 1973, vol. I, pp. 64-65 (Cixila, Vita vel gesta sancti Ildefonsi). Le récit est ensuite repris et véhiculé aux XI XII ${ }^{\mathrm{e}}$ siècles par toute la tradition des miracula.

24. S. Barnay, Le ciel sur la terre, op. cit., pp. 43-45.

25. Jourdain de Saxe. Libellus de principiis ordinis praedicatorum, éd. H. C. Scheeben, Rome, 1935, p. $52, \mathrm{n}^{\mathrm{e}} 57$.

26. Vita auctore Theodorico de Appoldia, AASS Aug. I, pp. 579-580 § 114-115-116. La vision des Miracula Beati Dominici a été éditée par A. Walz, « Die Miracula Beati Dominici der schwester Cäcilia. Einleitung und Text ", Miscellanea Pio Paschini. Studi di Storia ecclesiastica, Rome, 1948, p. 317 et P. I. Taurisano, Fontes selecti Vitae S. Dominici de Guzman. II. Miracula S. Dominici Romae patrata e a Beata Cecilia romana descripta, Rome, p. 48.

27. Respice Stellam. Maria in San Bernardo et nella tradizione cistercense, dir. I. M. Calabuig, Rome, 1993.

28. Thomas de Cantimpré, De Apibus, Douai, 1627, II, 10, 16, p.170. Cité également par J. Delumeau, Rassurer et protéger, op. cit., pp. 268-269.

29. Thomas de Celano, Vita II, chap. $150 \S 198$, cité par T. Desbonnets et D. Vorreux, Saint François d'Assise. Documents, écrits et premières biographies, Paris, 1968, p. 490.

30. Analecta franciscana sive chronica aliaque varia documenta ad historiam fratrum minorum edita a patribus collegii S. Bonaventurae, t. III, Quaracchi, 1897, p. 679.

31. Sur le contexte d'apparition du motif iconographique de la Vierge de miséricorde, voir notamment C. Belting-Ihm, op. cit., p. 70. Le plus ancien motif iconographique retrouvé figure sur les bannières de la Confraternitas Commendatorum Virgini fondée à Rome en 1267.

32. Pour la tradition servite, voir notamment $L a$ «Legenda de origine Ordinis fratrum Servorum Virginis Mariae" dei Servi di Maria. Testo latino et traduzione italiana, éd. de D. Pieraccioni et E. Toniolo, Roma, 1982, cap. XIII, p. 219. Pour la tradition carme, voir notamment Speculum Carmelitanum sive Historia Eliani Ordinis Fratrum Beatissimae Virginis Mariae de Monte Carmelo, Anvers, 1680, p. 117 et B.F.M. Xiberta, De visione Sanctis Simonis Stock, Rome, 1950.

33. L'expression est de D. Iogna-Prat, E. Palazzo, D. Russo, Marie. Le culte de la Vierge dans la société médiévale, Paris, Beauchesne, 1996, p. 7.

34. Cité par J. Delumeau, Rassurer et protéger, op. cit., p. 264.

35. E. Follieri, «La Theotokos difesa del popolo cristiano nelle tradizioni bizantine et italogreche », op. cit., pp. 377-383.

36. S. Barnay, Le ciel sur la terre, op. cit., chapitre III.

37. Ibid. 
38. H. Isnard, « Recueil des miracles de la Vierge du XIII ${ }^{\mathrm{e}}$ siècle », Bulletin de la Société archéologique $d u$ Vendomois, 26, 1887, pp. 36-38. On trouve une version très proche de ce texte dans T.F. Crane, Liber de miraculis sanctae Dei Genitricis Mariae, Vienne, 1731, reprint Londres, 1925, p. 45. Un exemple de la Vierge protectrice des naufragés est rapporté par exemple dans A. Meister, «Die Fragmente der libri VIII miraculorum », Römische Quartalschrift, Rome, 1901, p. 166.

39. Sur l'imitation de la Vierge au XIII ${ }^{\mathrm{e}}$ siècle, S. Barnay, Le ciel sur la terre, op. cit., chapitre III.

40. Vita Gerardescae Pisis, AASS Mai VII, p. 171, § 44.

41. Ibid.

42. A. Dondaine, «Barthélémy de Trente», Archivum Fratrum Praedicatorum, 45, 1975, $\mathrm{n}^{\circ} 56$, p. 95-96.

43. J. Delumeau, Rassurer et protéger, op. cit., pp. 261-289.

\section{AUTEUR}

\section{SYLVIE BARNAY}

Université de Metz, Institut catholique de Paris 